\title{
Test System of Electric Vehicle Charging Interface Based on Standard Command for Programmable Instruments
}

\author{
Guang Chen ${ }^{1, ~ a}$, Liang Xü ${ }^{1, b}$, Jing Zhang ${ }^{2, ~ c}$, Jing Zhang ${ }^{2, d}$ and Chang Liu ${ }^{2, e}$ \\ ${ }^{1}$ Beijing Sg-epri UHV Transmission Technology Co., Ltd, Beijing 102208, China; \\ ${ }^{2}$ China Electric Power Research Institute, Beijing Electric Vehicle Charging/Battery Swap \\ Engineering and Technology Research Center, Beijing 100085, China. \\ a chenguang@sgepri.sgcc.com.cn, b computerxl@163.com, 'c18610932703@163.com, \\ dopkl_5606@163.com, 'liuchang3@epri.sgcc.com.cn
}

Keywords: electric vehicle charging interface, conformance test, acquisition of electric signal

\begin{abstract}
SCPI protocol for acquisition of electric signal of electric vehicle charging interface with high accuracy, high speed and automation, analyzes current difficulty in conformance test of electric vehicle charging interface, designs a conformance test system of electric vehicle charging interface, introduces system structure, working flow and conformance test standard of the system in detail and analyzes long-term benefits of the system.
\end{abstract}

\section{Introduction}

At present, electric vehicle industry has been listed in seven strategic emerging industries in China. However, one of important difficulties under such fast development situation is that in large construction scale of charging facility network, many manufacturers adopt different charging piles and have different standard compliant degrees, which generates great dilemma for coverage and interconnection of whole charging network.

Viewing from electric vehicle users, users cannot charge in different charging equipment. Charging facilities cannot meet charging demand and user experience. Promotion and application of electric vehicle are severely obstructed and charging facilities are wasted greatly. Therefore, with increasingly improvement of industrialization degree of electric vehicle and fast promotion of construction of charging infrastructures, conformance and conformance test of charging interfaces receive great attention of vehicle industry, power industry and other related industries at home and aboard.

Research of conformance test technology of electric vehicle charging interface, research and production of conformance test device and system of electric vehicle charging interface, test and standardization of charging equipment interfaces of different manufacturers and solution of compatibility and communication protocol conformance problem of electric vehicle charging interface lay the solid foundation for further promotion of electric vehicle.

This paper introduces a conformance test system of electric vehicle charging interface based on SCPI protocol measuring instrument [1]. The system realizes automatic acquisition and automatic control of electric signals of electric vehicle charging interface based on interface interconnection requirement specified in national standard and by program control means, data real-time acquisition and other information technologies and meets automatic test requirement by comparison of acquired data.

\section{General Framework of Test System of Electric Vehicle Charging Interface}

System Hardware Components. The system is mainly composed of test interface, test control board, oscilloscope, test computing host and display. The core components are test control board, oscilloscope and test computing host [2].

Schematic diagram of system hardware is as follows. 


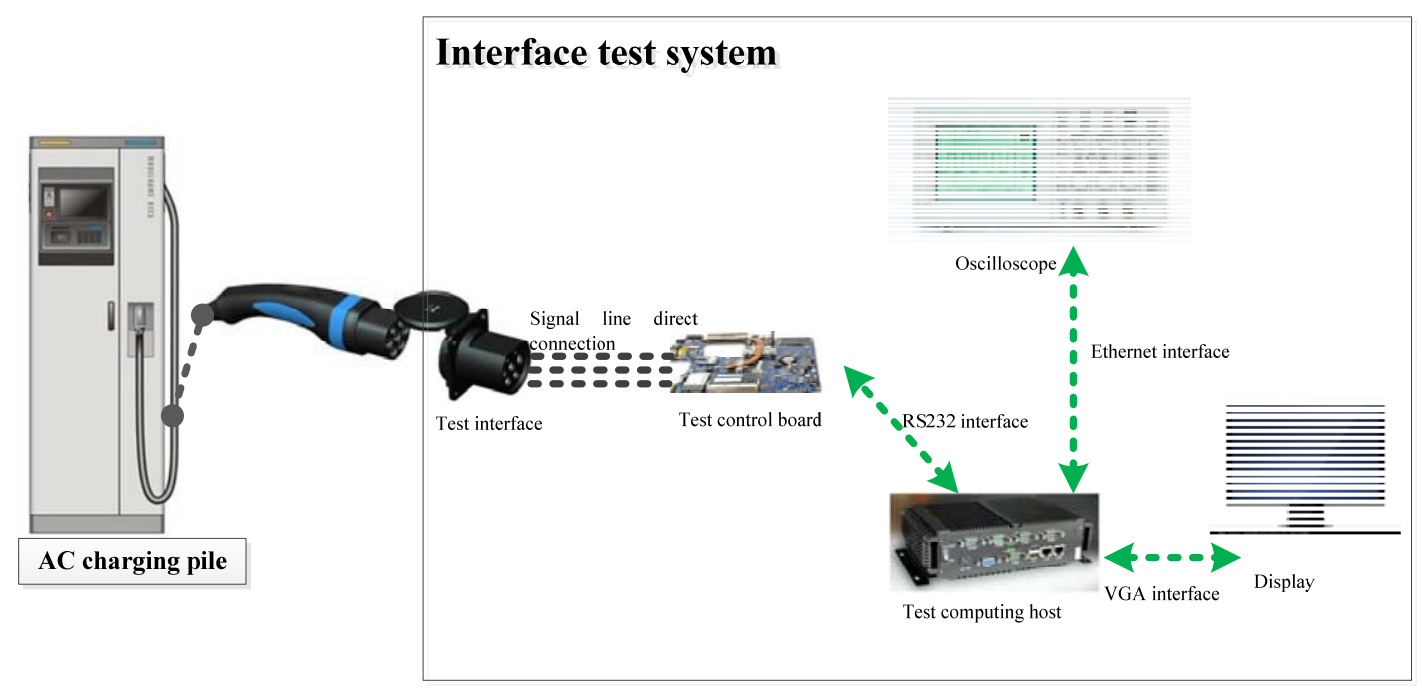

Fig. 1 Schematic diagram of system hardware

- Main components:

1. Test interface

It is the only interface connecting the tested charging facility and the test system. The tested electric signal is connected with the test system via the interface.

2. Oscilloscope

It can test the change of waveform of test points, pwm parameter, timing record and state of main loop contactor.

3. Test computing host

It can control oscilloscope and test control board, implement the test item, record and analyze the test result and provide human machine interaction interface.

4. Test control board

It can simulate the electric vehicle, control the internal line, sampling point and sampling time of test instrument and conduct conformance test of communication protocols.

5. Display

It can show process, real-time data and result of the test.

System Software Composition .The system software is mainly composed of two parts.

- Test control board software

Control board software implements control of every test item flow. Data interchange between the test control board and the test computing host is based on MODBUS communication protocol, the data include test communication message, control information of switches, status information of switches. The test control board software realizes switch control, oscilloscope signal acquisition and synchronous control of data reading of test computing host.

- Test computing host software

Test computing host software, as the core of the whole system, can finish set of all test items, provide human machine interface and provide test result output report. Data interchange between the test computing host software and the control board is based on MODBUS communication protocol. It can acquire sampling data of interface test electric signals from oscilloscope via standard command for programmable instruments (SCPI). It can, based on test flow, carry out computation of acquired data, finally provide test result and display in human machine interface. Schematic diagram of software flow is as follows. 


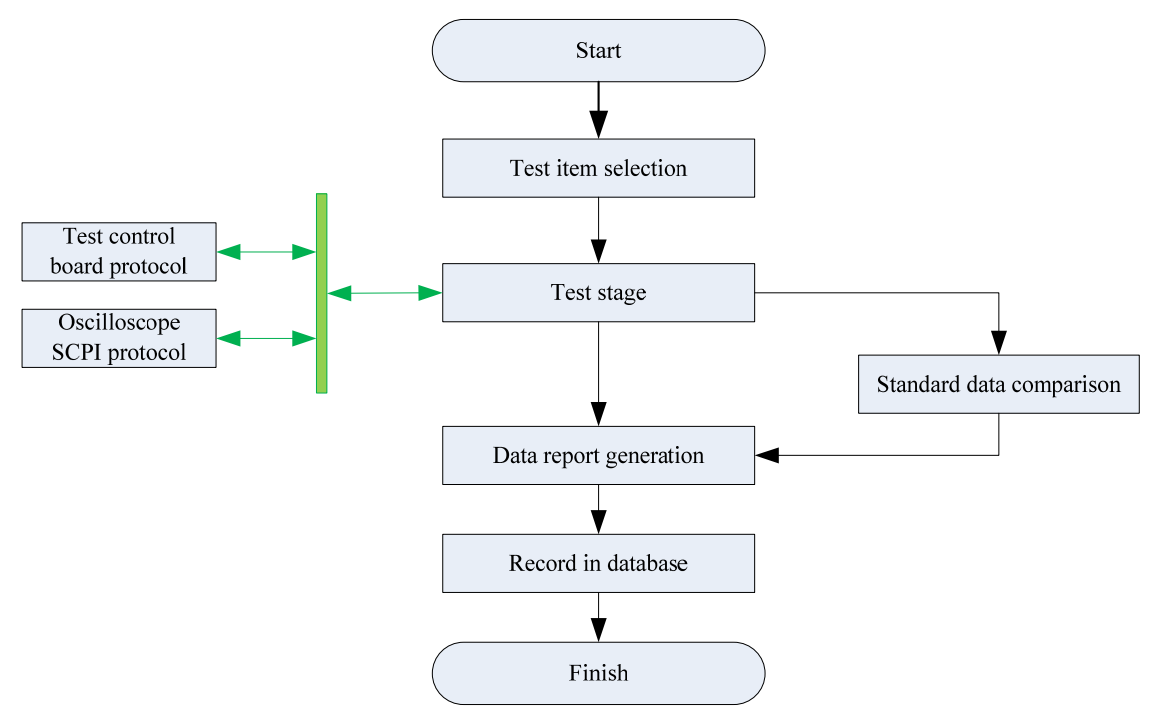

Fig. 2 Schematic diagram of software flow

\section{Software Design of Test System of Electric Vehicle Charging Interface [3]}

Software of test system of electric vehicle charging interface is operated in the test computing host, controls flow and time sequence of test and meets the requirement of every test item for evaluating test points and voltage, current and time sequence in charging loop. The system can control parameters of oscilloscope and read the data acquired by oscilloscope through establishing the data communication link based on SCPI protocol. Therefore, it realizes automatic test and improves current low efficiency situation caused by much manual interference in interface test activities.

Function of System Software. The software mainly has the function of test item management, test parameter setting, test data inquiry, test basis process and test result judgment and so on.

- Test item management: conduct interface test item management of AC charging pile, including test item addition, test detail, report output and distribution of test flow.

- Test parameter setting: provide interface for users and provide custom test items. The software saves the custom test parameters and permits quote of subsequent test.

- Test data inquiry: inquire the historic equipment test situation and show to users by human machine interface.

- Test basis process: treat the synchronization event of control board and test host, read the acquired electric signal data from oscilloscope in every test stage based on synchronization event and carry out data recovery in the system.

- Test result judgment: according to a series of related national standards: $\mathrm{GB} / \mathrm{T}$ 20234.1-2015 Connection Set for Conductive Charging of Electric Vehicles, GB/T 20234.2-2015 Connection Set for Conductive Charging of Electric Vehicles, GB/T 20234.3-2015 Connection Set for Conductive Charging of Electric Vehicles, GB/T 18487.1-2015 Electric Vehicle Conductive Charging System, GB/T 27930-2015 Communication Protocol between Off-board Conductive Charger and Battery Management System for Electric Vehicle, Interoperability Test Specification of Electric Vehicle Conductive Charging, synchronously read test record data is compared with standard requirements, the test result data is judged and the result is displayed on human machine interface.

Design of Test Software based on SCPI Protocol [4]. The oscilloscope can communicate through Ethernet bus and test computing host and carry out programming control based on SCPI (Standard Commands for Programmable Instruments), i.e. standard command set of programmable control instrument (programmable instrument). SCPI is a kind of standardized instrument programming language which is established based on existing standards IEEE 488.1 and IEEE 488.2 and follows floating point arithmetic rule in IEEE 754, ISO 646 information exchange 7-bit 
code symbol (equivalent to ASCII programming) and other standards. SCPI has the tree structure and includes many subsystems. Every subsystem is composed of one root keyword and one or many hierarchical keywords.

Flow of interface connection performance test of AC charging pile is as follows [5]:

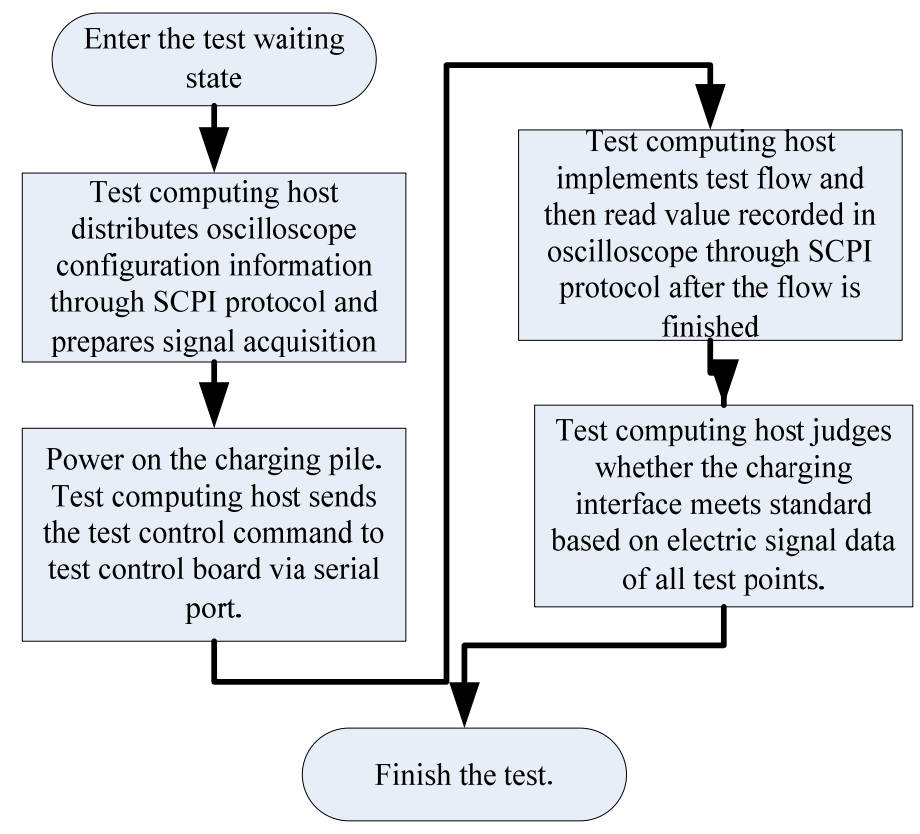

Fig. 3 Flow of interface connection performance test of AC charging pile

1) Prepare the test environment. Power on the tested charging pile and test system. Ensure the equipment in standby state;

2) Select the test item in test item interface of test computing host and start the test flow. Test computing host connects with oscilloscope by Ethernet, sends the oscilloscope channel, signal interception and other operation and configuration information packed based on SCPI standard format to oscilloscope and realizes automatic and programmed operation of oscilloscope;

3) After connecting the test equipment and setting the operation mode, start the test flow. The test computing host decomposes the item to be tested into operation sequence and implements based on sequence. During implementation of test item, the switch control operation shall be conducted. The host sends the control command to the test control board through serial port to control the test switch and realize automatic and programmed test flow.

4) Electric signal of tested equipment is acquired and stored by oscilloscope and other instruments. The host packs the test point reading operation parameter based on SCPI protocol and sends to oscilloscope. After data reading, the host analyzes the data of sampling point based on SCPI protocol and calculates test result based on relevant standards [6];

\section{Operation Practice of Test System of Electric Vehicle Charging Interface}

Take A1.3502 as an example to show the effect of test operation of the system.

1) Select "AC test" -> "abnormal charging test"->"CP open circuit test" on test interface of test computing host.

2) Select "start test". The test process will automatically perform the following operations: After fully connecting the charging connection device, start charging. After 10s, disconnect the CP line by simulation and test the PWM signal of CP line and signal of charging plug. 


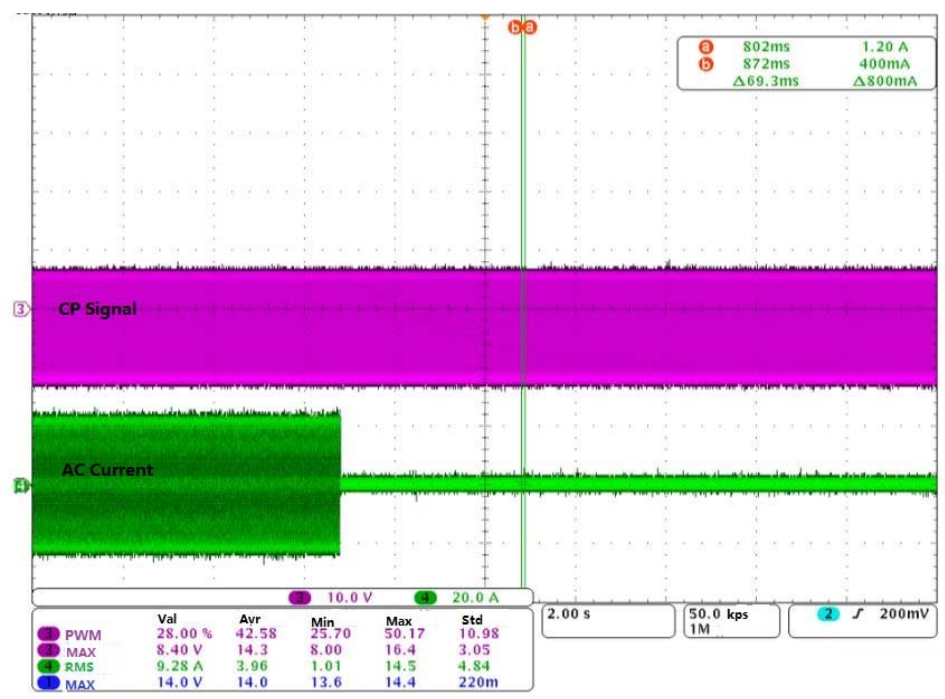

Fig. 4 The relationship of CP signal and AC signal during charging process

3) Judge whether the test item passes based on signal data.

It is specified in GB/T 18487.1-2015 that during charging, after CP signal is disconnected, the AC power supply shall minimize the current within $100 \mathrm{~ms}$. The tested equipment minimizes the current in $180 \mathrm{~ms}$ after the disconnection of CP signal. Therefore, the test item cannot pass.

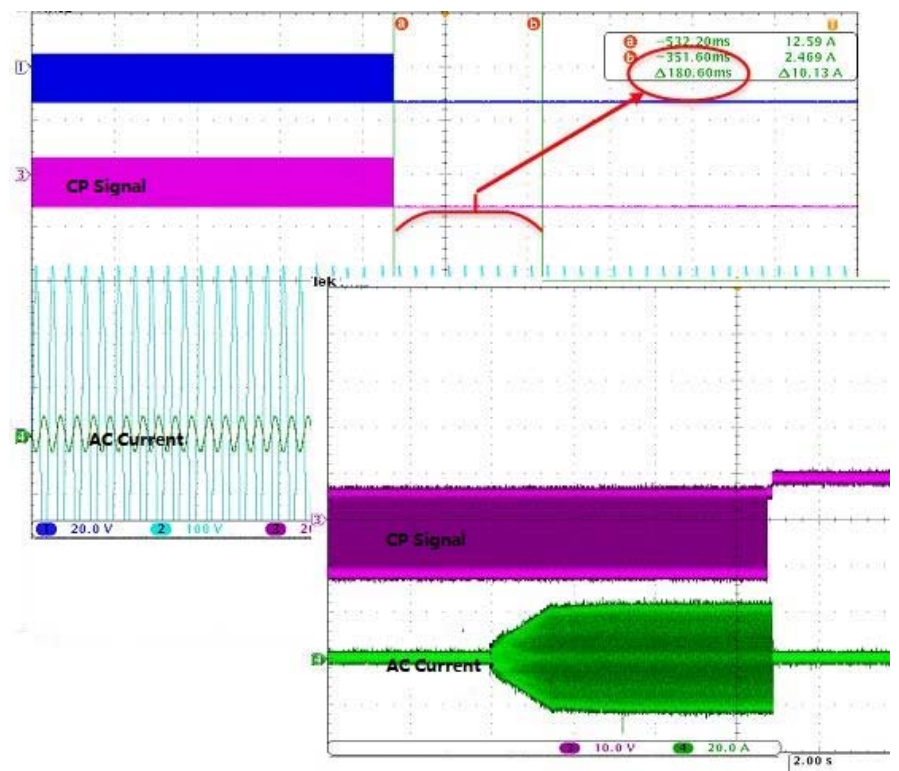

Fig. 5 The reaction of AC signal when CP simulated signal is fault

\section{Conclusions}

This paper introduces system structure, software and hardware composition and software function of test system of electric vehicle charging interface based on Standard Command for Programmable Instruments in detail and focuses on design ideas and realization method of test system based on SCPI protocol. Finally, the test system is used to test the AC charging pile to verify the availability and reliability of the test software. The test system based on SCPI protocol will promote standardization of charging interfaces of charging facility, improve automation level of interface test and contribute to improving quality of charging equipment of electric vehicle.

\section{Acknowledgment}

This work was supported by one of science and technology project of State Grid Corp of China named Key technology and verification of the consistency testing of AC/DC charging interface of 
electric vehicle.

\section{References}

[1] Standard Commands for Programmable Instruments Manual.1990.

[2] Texas Instruments incorporated, SN75160,May 1995

[3] ChenJinping, WangShengze,WuWenying, Application of LabVIEW Event Drives in Menu Design, 《Instrumentation Technology》, 2005(1):13-14

[4] FJ Jimenez, JD Frutos, Virtual instrument for measurement, processing data, and visualization of vibration patterns of piezoelectric devices, 《Computer Standards \& Interfaces》,2005

[5] A Yazidi, H Henao, GA Capolino,A Web-Based Remote Laboratory for Monitoring and Diagnosis of AC Electrical Machines, 《IEEE Transactions on Industrial Electronics》,2011

[6] X Kong, Y Li, L Chen, The Application of a New Embedded Virtual Instrument System, World Congress on Intelligent Control \& Automation,2008 